\title{
The Circulating Biomarkers Concentration and Endoleak Monitoring After Stentgraft of Abdominal Aorta
}

\author{
Antonio Bozzani* \\ Vascular Surgery Unit, Foundation I.R.C.C.S. Policlinico San Matteo, Pavia, Italy
}

\begin{abstract}
Abdominal aortic aneurysm (AAA) is a common degenerative condition with an estimated incidence of 20 to 40 cases per 100,000 , and nearly 45000 surgical operation are performed annually in the United States for the treatment of this potentially lethal disease. Nowadays endovascular techniques comprise the mainstay treatment of AAA in patients with suitable anatomy. Randomized trials have demonstrated a perioperative survival benefit associated with endovascular AAA repair (EVAR) compared with open repair. There are concerns, however, about the intermediate and longerterm outcomes, with EVAR being associated with increased risk of reintervention. Endoleak $(E L)$ is the most common complication after infrarenal aneurysm EVAR with a reported incidence varying from 2 to $45 \%$ associated with sac pressurization, aneurysm growth and increased risk of rupture at 1-year follow up [1-3]. Computed tomography angiography (CTA) is considered the gold standard for the detection of EL, despite known disandvantages such as cumulative radiation risk, nephrotoxic contrast agent and high costs. Therefore a less harmful and cost effective alternative surveillance method for follow-up is desirable. Contrast enhanced ultrasonography (CEUS) is a valuable less invasive and cost effective alternative with high sensibility and specificity. A meta-analysis showed an accumulated specificity of $98 \%$, respectively a sensitivity of $88 \%$, for the finding of an endoleak. The accuracy of CEUS in the recognition and classification of endoleaks after EVAR seems to be high, as recently demonstrated prospectively with a sensitivity of $97 \%$, a specificity of $100 \%$ and an accuracy of $99 \%$ [4-6].
\end{abstract}

Furthermore, it is postulated that the continued perfusion of the aneurysmal sac in patient with endoleak perpetuates the aortic wall remodelling resulting in circulating biomarkers release. Matrix metalloproteinases (MMPs) play a pivotal role in the integrity and composition of the extracellular matrix, whose major components are collagen and elastin. Increased MMP-mediated collagenolytic and elastolytic activity is present in the aneurysmal aortic wall. An imbalance between MMPs and their inhbitors (tissue inhibitors of MMPs [TIMPs]) impairs normal physiological aortic wall remodelling and is integral to aneurysm development. Nowadays few studies, with a total patient sample around 130, have explored the relationship between imaging and circulating biomarkers fluctuation during time.

Under normal physiological conditions, the turnover and remodeling of the extracellular matrix is regulated by proteolytic mechanisms, whereas a dysregulation in these system contributes to abnormal collagen metabolism and the generation of patologic conditions, such as abdominal aortic aneurysms (AAAs). MMPs play a pivotal role in the integrity and composition of the extracellular matrix, whose major components are collagen and elastin. The MMPs and their naturally occurring inhibitors are biochemical markers of extracellular matrix degradation and remodelling. Patients with AAA have elevated circulating MMP-9 levels compared with control individuals [7]. Emerging evidence suggests that persistently raised circulating concentrations of MMPs in patients having previously undergone EVAR reflect a continuing degrading process of the aortic wall, which results from inadequate aneurysm exclusion. Successful stent graft treatment of the AAA results in the inhibition of such precipitating events, whereas inadequate aneurysm exclusion in the presence of an endoleak leads to an ongoing pathogenic process resulting in propagating degrading mechanisms, aneurysm expansion, and rupture. Theoretically, increased aortic wall extracellular matrix inflammatory and degrading activity results in a continuous release of soluble MMPs in the circulation. Therefore, circulating MMP levels may serve as a marker of ongoing disease in patients with persistent endoleak following EVAR. Persistently elevated plasma levels of MMPs after EVAR may discriminate between patients with and without an endoleak. Establishment of a reliable blood test for the detection of endoleaks may reduce the need for CTA in the follow up of patients undergoing EVAR $[8,9]$.

\section{REFERENCES}

1. Drury D, Michaels JA, Jones L, Ayiku L. Systematic review of recent evidence for the safety and efficacy of elective endovascular repair in the management of infrarenal abdominal aortic aneurysm. Br J Surg. 2005;92(8):937-46.

Correspondence to: Antonio Bozzani, Vascular Surgery Unit, Foundation I.R.C.C.S. Policlinico San Matteo, Pavia, Italy, Tel: +390382502068; E-mail: a.bozzani@smatteo.pv.it

Received: February 26, 2019; Accepted: March 29, 2019; Published: April 05, 2019

Citation: Bozzani A (2019) The Circulating Biomarkers Concentration and Endoleak Monitoring After Stentgraft of Abdominal Aorta. J Vasc Med Surg 7:382. doi: 10.35248/2329-6925.19.7.382

Copyright: (C) 2019 Bozzani A. This is an open-access article distributed under the terms of the Creative Commons Attribution License, which permits unrestricted use, distribution, and reproduction in any medium, provided the original author and source are credited. 
2. Arici V, Quaretti P, Bozzani A, Moramarco LP, Rossi M, Carlino M. Neck-targeted, stand-alone coiling for successful treatment of type 1A endoleak following endovascular repair. Vasc Endovascular Surg. 2014;48(1):61-4.

3. Bozzani A, Arici V, Ragni F, Argenteri A. Endoleak after endovascular aortic repair and lumbar vertebral erosion. J Orthop Traumatol. 2015;16(1):75.

4. Arici V, Perotti C, Fabrizio C, Del Fante C, Ragni F, Alessandrino F, et al. Autologous immuno magnetically selected CD133+ stem cells in the treatment of no-option critical limb ischemia: clinical and contrast enhanced ultrasound assessed results in eight patients. J Transl Med. 2015;3(13):342.

5. Perotti C, Arici V, Cervio M, Del Fante C, Calliada F, Gnecchi $\mathrm{M}$, et al. Allogeneic lethally irradiated cord blood mononuclear cells in no-option critical limb ischemia: a "box of rain". Stem
Cells Dev 2013;22(20):2806-12.

6. Bozzani A, Arici V, Bonalumi G, Argenteri A. Descending thoracic aorta remodeling after multilayer stent release. Ann Vasc Surg. 2015;29(5):1018.e9-1018.e11.

7. Arici V, Bozzani A, Odero A. Successful endovascular treatment of a bronchial artery aneurysm refractory to transcatheter embolization. Ann Vasc Surg. 2013;27(6):802.e13-5.

8. Golledge J, Tsao PS, Dalman RL, Norman PE. Circulating markers of abdominal aortic aneurysm presence and progression. Circulation. 2008;118(23):2382-92.

9. Ng E, Morris DR, Golledge J. The association between plasma matrix metalloproteinase-9 concentration and endoleak after endovascular aortic aneurysm repair: a meta-analysis. Atherosclerosis. 2015;242(2):535-42. 